\title{
Los planos de colonización de Cautín y Malleco (1916-1917): expresión de la morfología de la Araucanía
}

\author{
The colonization maps of Cautín and Malleco (1916-1917): Exposing the morphology of Araucanía
}

\author{
Wladimir Antivil Marinao \\ Facultad de Arquitectura, Artes y Diseño. Universidad Católica de Temuco, Temuco, Chile \\ wantivil@gmail.com
}

\begin{abstract}
Resumen
El siguiente trabajo pretende explorar y poner en valor los planos catastrales de colonización hechos para dos provincias de Chile, llamadas Cautín y Malleco, los años 1916 y 1917 respectivamente. Dichos planos son fruto de la intervención física iniciada por el Estado de Chile durante la segunda mitad del siglo XIX la cual tuvo como fin someter esos territorios a la soberanía nacional. El trabajo plantea que estos planos logran esclarecer los sucesos ocurridos en todo el proceso con un nivel de detalle importante presentando gran cantidad de datos. Además, plantea que, si se estudian los dos planos en conjunto, a pesar de que el área que cubren es extensa, es posible interpretar las intenciones físicas y espaciales que se intentaron instalar sobre el territorio, especialmente se puede apreciar una idea y estructura espacial que lo ordena en la dirección norte sur. El trabajo se desarrolla aplicando un planteamiento metodológico con énfasis en el estudio de la morfología, el cual consiste en identificar los componentes dibujados de los planos, observar sus repeticiones, redibujarlos y encontrar relaciones espaciales entre los componentes. Se ha considerado también el estudio de algunos documentos y antecedentes bibliográficos.
\end{abstract}

Palabras clave: Araucanía. cartografía, colonización, morfología.

\begin{abstract}
The following work explores and highlights the significance of the cadastral colonization maps made in 1916 and 1917 for two provinces of Chile: Cautín and Malleco, respectively. These maps were the result of colonization planning initiated by the State of Chile during the second half of the nineteenth century, an exercise which sought to bring the two territories under national control. The maps are highly detailed and contain a substantial amount of data, and we propose that they offer considerable insight into the colonization process. We also posit that, although the area covered is extensive, a study of the two maps together would allow us to interpret the physical and spatial nature of the territorial interventions envisaged by the State and, in particular, offer insights into the intended north-south spatial structure of the region. Our methodological approach focuses on the morphology of the maps and involves a study of their drawn components, identification of any repeated configurations, tracing work, and examination of spatial relationships between components. A number of other documents and bibliographic references were also studied.
\end{abstract}

Keywords: Araucanía, cartography, colonization, morphology.

Documento recibido el 23 de diciembre de 2019 y aceptado el 29 de mayo de 2020

Este trabajo es fruto de una tesis doctoral la cual fue desarrollada gracias a Becas Chile, Becas de doctorado en el extranjero, CONICYT.

Cómo citar: Antivil, W. (2020). Los planos de colonización de Cautín y Malleco (1916-1917): expresión de la morfología de la Araucanía. Revista de Urbanismo, 42, 134-150. https://doi.org/10.5354/0717-5051.2020.55710 
Cautín y Malleco son dos provincias que componen la actual Araucanía, la cual ha sido un territorio complejo a lo largo de la historia de Chile ${ }^{1}$. En la segunda mitad el siglo XIX dicho territorio es incorporado definitivamente a la soberanía del Estado chileno a través de diversos medios como el avance de fuerzas militares, la labor de ingenieros agrimensores y algunas iniciativas políticoadministrativas, entre otros. La acción de estos medios generó una transformación física del territorio la cual se fue generando en un proceso continuo, pero con diversos momentos, lugares y personal técnico. Toda esta transformación queda registrada en algunas cartografías de la época, especialmente en los planos de estas dos provincias hechos en el año 1916 para el caso de Cautín y 1917 para el caso de Malleco. Durante dichos años los procesos de dominio chileno sobre la Araucanía ya habían avanzado prácticamente en todo el territorio por tanto estos planos reflejan la parte final de dichos procesos.

El presente artículo tiene por objetivo, en primer lugar, exponer algunos conocimientos que pueden entregar estos planos sobre la forma que adquiere el territorio de la Araucanía. En ese sentido, se busca rescatar el valor propio de éstos. En segundo lugar, pretende reflexionar sobre la forma que adquiere el territorio bajo una metodología de análisis territorial con fuerte carga interpretativa hacia los dibujos y planos. Ante aquellos objetivos surgen dos preguntas ¿Qué conocimientos o valores aportan estos planos? ¿Qué reflexiones, en cuanto a la forma o idea espacial, nos dejan en la gran escala?

El trabajo plantea que los planos catastrales de colonización de la Inspección General de Colonización e Inmigración (en adelante $I G C I$ ) para las provincias de Cautín y Malleco ${ }^{2}$ constituyen una imagen que refleja y aclara lo ocurrido durante todo el proceso de conquista y colonización chileno sobre la Araucanía. Además, dichos planos permiten identificar algunos componentes morfológicos, tanto naturales como construidos que se explicarán más adelante. El valor de los planos está en la cantidad de datos que entregan, en su destacable

\footnotetext{
${ }^{1}$ Ha sido complejo porque históricamente se han registrado numerosos conflictos por su dominio. Desde el siglo XVI se conocen los enfrentamientos entre las agrupaciones mapuches y los colonizadores españoles. Con el paso del tiempo, de acuerdo a las cartografías coloniales, se observa que los colonizadores optaron por replegar la frontera física de la colonia hasta el río Biobío. Más tarde, cuando Chile se independiza, el naciente estado chileno se encuentra con un territorio sobre el cual se había impedido la construcción
}

capacidad de interpretación y en que son expresión bastante acertada de los procesos de dominio chileno. Con los antecedentes revisados parecen ser los planos más completos de todo el proceso de colonización chilena en la Araucanía, y eso no es menor, ya que en dicho periodo se elaboró una gran cantidad de planos para dicho territorio.

El otro planteamiento de este trabajo es que estos planos aportan unas ideas espaciales de dominio y construcción del territorio. La forma en que se parcela el suelo rural junto con otros componentes de los planos permite ver intenciones en la escala regional donde se aprecia la importancia de generar una continuidad espacial de norte a sur. Para esto el trabajo sigue una metodología orientada principalmente a la morfología y construcción del territorio, donde las repeticiones morfológicas de los componentes del plano son importantes para la interpretación de los planos en la escala regional. El resultado nos lleva a descubrir un proceso complejo dado en un marco de tiempo, que involucró una suma de procesos que tuvieron su expresión física sobre el territorio; tales procesos se gestaron unos sobre otros, en tiempos sucesivos y distintos, y no solo en una trabajada implantación sistemática y homogénea.

La elaboración de estos planos estuvo a cargo de Nicanor Boloña quien, todo indica, confecciona estos grandes planos de colonización a partir de planos más locales que abarcan zonas de dimensión más acotadas.

Existe una creciente cantidad de trabajos sobre las relaciones entre cartografía, ciudades y territorios chilenos. En dichos trabajos la cartografía es fuente fundamental (Capellà, 2009; Chamorro y Urbina, 2018; Figueroa, 2011; González \& Bernedo, 2013; Hidalgo, Rosas, \& Strabucchi, 2012; Rosas, Hidalgo, Strabucchi y Bannen, 2015). También existen trabajos sobre los modos de proceder en la elaboración de los inicios de la cartografía nacional (Álvarez, 2000; González, 2007), así también un destacable trabajo sobre cartografía histórica nacional (Rosenblitt y Sanhueza, 2010).

\footnotetext{
de ciudades y que se encontraba en la mira para saber qué realmente se debería hacer sobre él.

${ }^{2}$ Las provincias de Malleco y Cautín conforman hoy la región de la Araucanía. Si bien al momento de hacer estos planos los límites de estas provincias no son exactamente los mismos que abarcan al día de hoy.
} 
Sumado a lo anterior, empieza a haber un interés en la cartografía de la Araucanía (Antivil, 2018; Bisbal-Grandal, 2019; Flores y Azócar, 2017; Gonzaléz \& Llancavil, 2017). Estas propuestas indirectamente plantean la capacidad de la cartografía como generadora de conocimiento y reveladora de realidades aún ocultas en dicho territorio. En el caso de González y Llancavil (2017) el interés está en la cartografía elaborada por misioneros. En el caso de Bisbal-Grandal (2019) su interés está en la cartografía colonial y de conquista española. Flores y Azocar (2017), y Antivil (2018), por otro lado, se interesan por el período de la República de Chile y ambos utilizan para su reflexión los planos de la IGCI. Flores y Azocar (2017) los usan, pero no de forma exclusiva sino en conjunto con otros mapas que manda a hacer el Estado de Chile en esa época. Antivil (2018), en tanto, usa los planos más bien como herramienta, también en conjunto con otras cartografías, para comprender su objeto principal que era el territorio de la Araucanía en sí. Por último, de forma anexa, también nos encontramos con un trabajo con foco en la ciudad de Temuco, donde una de las primeras cartografías urbanas ayuda a la comprensión de algunos fenómenos sociales (Rojo y Hernández, 2019).

El presente trabajo considera como antecedente importante los trabajos de Flores y Azocar (2017), y especialmente el de Antivil (2018) como base, aunque en este caso, el trabajo busca diferenciarse porque intenta reconocer el valor propio de los planos de la IGCI. Dichos planos son un insumo y fuente de datos de incalculable valor para empezar a comprender los complejos procesos espaciales dados en el origen de esta región.

Se debe señalar que el presente trabajo no pretende una aportación teórica, sino más bien, a través de un método de análisis, entregar conocimiento sobre un territorio específico, en este caso la Araucanía.

Un trabajo que pone su mirada en el territorio debe considerar el modo de estudiarlo y, como primer tema, surge el de la escala a estudiar. Para el caso de este trabajo consideramos el estudio de la escala regional de tal modo que sea posible extraer algunas ideas o principios de ordenación general que se logran observar en todo este

\footnotetext{
${ }^{3}$ Los planos de la IGCl también evidencian, en parte, algunos patrones morfológicos que pueden ser fruto de una perspectiva mapuche. Esto debido a que se pueden ver con bastante detalle cómo quedaron distribuidas gran parte de las tierras mapuches. Creemos que la variable mapuche debiese
}

proceso de colonización de las dos cartas provinciales. Explicitamos esta perspectiva conscientes de que existen otras escalas de aproximación y análisis territorial.

Como su nombre lo indica, son planos de colonización; esto quiere decir que tienen un autor intelectual y ese autor ya tiene una visión parcial del territorio. Los planos estudiados, por tanto, lejos de ser objetivos respecto a una realidad, más bien expresan una visión principalmente desde la entidad colonizadora ${ }^{3}$.

Para comprender mejor el tema a estudiar, antes de exponer los resultados, se exponen breves antecedentes necesarios para comprender el proceso de ocupación de la Araucanía. Luego se explican aspectos metodológicos del trabajo usando un método de análisis el cual considera la identificación de componentes morfológicos del territorio, tales como los factores naturales y elementos construidos. Considera también las observaciones que se pueden deducir a partir de las repeticiones y las relaciones entre dichos componentes.

Los resultados llevan a plantear tres temas. Primero, la descripción de los planos y la capacidad que tienen para revelar algunos temas que empiezan a aparecer. Segundo, la importancia de las repeticiones o patrones morfológicos que revelan los componentes del plano. Tercero, la idea de continuidad espacial norte-sur que se refleja en los planos y que, planteamos, refleja la idea estructural de dominio chileno.

\section{Antecedentes para comprender la ocupación chilena en la Araucanía}

Durante la segunda mitad del siglo XIX el territorio al sur del Biobío fue sometido a la ocupación chilena. El Biobío, como ya se ha difundido por la historia, sirvió de frontera entre los dominios coloniales y los dominios mapuches. Independientemente de toda la discusión que exista en torno a cuánta autonomía real había en los territorios del interior de la actual Araucanía durante la época colonial, se observa un territorio sin mucha cartografía precisa y sin construcciones coloniales que permaneciesen. Algunas cartografías (Instituto Geográfico Militar y Medina, 1952; Rosenblitt \& Sanhueza, 2010, pp. 177-179) manifiestan

considerarse para una comprensión más cabal de estos planos. Sin embargo, para estudiarla se requiere de un esfuerzo adicional que debiésemos tratar en otro trabajo. Por ahora interesa aproximarnos a la visión de estos planos más bien desde la perspectiva colonizadora. 
que, si bien las zonas aledañas al Biobío estaban cartografiadas con cierto detalle y presentaban un número de construcciones importante para entonces, al sur del Biobío prácticamente no había construcciones y, si es que había, se señalan solo como pueblos o construcciones ya destruidas. Las construcciones aledañas al Biobío permanecieron hasta el surgimiento de la nación chilena, en tanto que los territorios al sur del Biobío permanecían en una situación inconclusa para el naciente gobierno 4 .

Desde los inicios de la República qué hacer con la Araucanía fue objeto de debate y de diversas ideas. Según lo señala Leiva (1984, p. 13), entre los años 1859 y 1862, al menos se propusieron diez ideas para reducir la Araucanía. Pinto (2015, p. 136), por otro lado, señala una serie de razones bastante orientativas acerca del porqué se lleva a cabo la ocupación de la Araucanía, señala factores externos e internos al país 5 . Junto a todo eso, se registran algunos relatos de viajeros y exploradores (Pinto, 2010, p. xiv) donde, al menos uno de ellos, Ignacio Domeyko, llegó a elaborar ideas para un posible avance del Estado sobre el territorio.

Es complejo atribuir a un solo documento, iniciativa o proyecto, la explicación del porqué de la ocupación chilena; lo que encontramos es, más bien, una suma de iniciativas y acciones por parte del Estado. Dichas acciones fueron de naturaleza diversa, tanto políticaadministrativa, militar y técnica. Desde lo político administrativo, como hecho significativo se crea la provincia de Arauco en 1852, la cual fue dividiéndose en diferentes zonas hasta convertirse en lo que hoy son las provincias de Malleco y Cautín (González y Bernedo, 2013). Desde lo militar, con las acciones e intenciones de

\footnotetext{
${ }^{4}$ Es interesante que, desde el siglo XVIII, se ven intenciones similares a las que pasan en época chilena del siglo XIX. Señala Gándara (2016, p.77) que "Las representaciones que los intelectuales ilustrados construyeron sobre este espacio giran en torno a dos ejes: primero, su importancia geoestratégica, y segundo, su potencialidad económica. En estos proyectos se desarrolla una interpretación de la frontera como un espacio problemático, por cuanto representa una fractura en el sistema territorial del Reino de Chile."

5 Cuando considera los factores externos, Pinto (2015, p.136) señala lo siguiente: "la mayor demanda de alimentos derivada del aumento de la población mundial y las transformaciones que provocó la Revolución industrial, estimularon la ocupación de regiones que hasta entonces se habían mantenido casi sin explotar". Entre los factores internos, el mismo autor (p.136), señala a la "estrechez del mercado de la tierra, esencial para sostener la política inmigratoria que se estaba fomentando desde el propio gobierno".
}

la llamada Pacificación de la Araucanía6, con enfrentamientos y avances de fuertes. Desde lo técnico con la elaboración de una serie de planos de exploración militar, de agrimensura y trazados que hicieron ingenieros, entre ellos Teodoro Schmidt. El avance sobre la Araucanía también fue de la mano con un desarrollo del aparato estatal y de las entidades a cargo de la colonización como la IGCl. Asimismo, el avance se logra con una numerosa cantidad de leyes y decretos.

Los hechos muestran que en el año 1862 el avance chileno se concreta con la fundación de Angol (Leiva, 1984). Luego, con la disposición de diversas líneas militares (Navarro, 2008), el avance llegará a ocupar todo el territorio de la Araucanía, lo que acabará con una Araucanía construida y dominada completamente por el Estado de Chile y con la aparición de diversas ciudades. Posteriormente el resto del territorio fue dominado por el Estado y sometido a una división del suelo que hasta entonces no existía. Dichas tierras sufren una serie de intervenciones de distinta naturaleza, algunas tierras son subastadas, otras entregadas tanto a colonias extranjeras como nacionales y, en otros casos, se les reconoce tierras a agrupaciones mapuches de entonces.

\section{Metodología}

El territorio es y ha sido objeto de estudio desde diversas disciplinas. En este caso, se considera una aproximación metodológica que se basa en el estudio de cartografía de ciudades y territorios, y la elaboración de planos interpretativos. En lo general, esta metodología proviene desde el trabajo de algunos arquitectos (De Solá-Morales y Parcerisa, 1981; Eizaguirre, 2019; González-Cebrián, 1984) relacionados con el Laboratorio de Urbanismo de Barcelona7 (en adelante LUB), los cuales han puesto su

\footnotetext{
Añade también como razón a la crisis económica de 1857, la revolución de 1859 y el discurso de la "barbarie" el cual estaba instalándose en grupos de poder de la época.

${ }^{6}$ Según Almonacid (2009, p.8), la llamada Pacificación de la Araucanía se sustentó en tres temas que se creían entonces, que también aparecen en otras fuentes. Primero, en que los indígenas que vivían ahí se encontraban en un estado de barbarie; segundo, el escaso número en que se encontraban dichos indígenas; tercero, que las tierras que ocupaban se encontraban en un estado de abandono.

${ }^{7}$ El Laboratorio de Urbanismo de Barcelona pertenece a la Escuela Técnica Superior de Arquitectura de Barcelona (ETSAB) donde se ha desarrollado una verdadera escuela de pensamiento para comprender e intervenir la ciudad y el territorio poniendo énfasis en los estudios morfológicos y en el proyecto
} 
atención a algunos territorios y ciudades españolas. Estos consideran el territorio como manufactura y sus observaciones tienden a basarse en la confianza por la consistencia de la forma. Así también, algunos de estos trabajos buscan interpretar las voluntades proyectuales de ciertos territorios poniendo mucha atención a la expresión gráfica de los planos. De algún modo apuestan a que la forma física de la construcción del territorio es el testigo último de los diversos procesos que lo afectan.

En un trabajo importante hecho por el LUB $^{8}$ se puede observar que es posible para los arquitectos analizar el territorio más allá de lo urbano. Se puede ver cómo usando algunas herramientas, que ya usaban para estudios morfológicos urbanos (caminos, parcelas, edificaciones y otras) con fuerte énfasis en el dibujo, fueron usadas para estudios del territorio. En dichos trabajos se puede observar también una mirada por encuadres específicos que incluyen construcciones urbanas y rurales, explicados de tal modo que no siguen la típica división clásica entre urbano y rural. En estos trabajos se ve también una intención claramente hacia el proyecto, hacia propuestas de proyectos urbanos y territoriales. Eso les da una doble virtud; por una parte, plantean ideas sobre intervención territorial y/o urbana, y por otra, plantean un buen método de estudio y análisis territorial, con atención a la morfología, intención y detalle de los planos.

Como valor del citado trabajo del LUB, más allá de las reflexiones escritas que produjeron está el hecho de la valiosa elaboración de cartografías propias que generaron. Tal como ellos el presente trabajo pretende aportar con la elaboración propia de cartografía a partir de los planos de la IGCl.

Para la presente investigación también se ha considerado fundamental el trabajo de Eizaguirre (2019), quien a su vez sigue el pensamiento de la escuela de Barcelona, Gregotti (1972) y escuelas italianas centradas en la morfología urbana. Desde su aproximación al

urbano y territorial. Esta mirada está influenciada también por escuelas italianas con énfasis en la morfología de las ciudades.

${ }^{8}$ Dicho trabajo estuvo centrado en estudios sobre las Comarcas de Cataluña el cual puede verse en el número extra 1 del año 1981 de la revista Quaderns d'Arquitectura i Urbanisme y en la web https://lub.upc.edu/index.html. El texto citado de Solá Morales y Parcerisa (1981) también es una muestra de territorio, la cual no pretende ser teórica sino más bien metodológica, se rescatan y se desarrollan dos ideas. En primer lugar, la idea de descifrar y entender los territorios a través de algunos componentes morfológicos dibujados en los planos, entre los cuales se distinguen, por una parte, factores naturales: cursos de agua y relieves, y, por otra, elementos construidos: caminos, división del suelo y edificación. En segundo lugar, la idea de las repeticiones de estos componentes como una clave para interpretar dichos territorios y plantear hipótesis de construcción territorial. Este método permite de algún modo comprender voluntades proyectuales detrás de la construcción de un territorio. Se trata entonces de un método bastante útil para acercarse a diversos territorios, sobre todo rurales. Una de sus principales virtudes es que no es un método cerrado, sino que puede agregar otros componentes, dependiendo del área geográfica a estudiar.

A partir de los trabajos del LUB observamos que una de las características de las repeticiones es que éstas evidencian diversas tipologías morfológicas. Esto es de importancia fundamental ya que manifiestan una identidad y cultura presente en el territorio, tanto en la acumulación de repeticiones como la escasez de ellas. La repetición, metodológicamente, es una de las claves para entender la forma, y se puede generar por diversas situaciones: en ocasiones es una medida parcelaria la que se repite, en otras simplemente la forma resultante de una relación marcada entre dos o más componentes del territorio. Si observamos los territorios rurales en otras latitudes se evidencia que existen repeticiones, las que son fruto más bien de una cultura territorial, de un modo de vivir, donde los habitantes usan el ejemplo del vecino para construir?

Se consideraron algunos pasos para estudiar los planos de la IGCl. El primero consistió en establecer como marco de estudio los dos planos (Malleco y Cautín) como un solo conjunto, esto lleva a centrar la observación en la escala dicho trabajo. Desde la citada web es posible ver otros trabajos territoriales del Laboratorio y también bibliografía de los trabajos de Manuel de SoláMorales.

${ }^{9}$ Esta idea aparece en Eizaguirre (2019, p.27), sin embargo, como se señala en el mismo texto la idea ya es planteada antes por Carlos Martí Aris, quien fue profesor de la Escuela Técnica Superior de Arquitectura de Barcelona. 
regional. Como segundo paso, se identifica la cantidad de componentes del plano; estos deben ser dibujables.

Siguiendo los métodos comentados, en los planos de la IGCl identificamos los cursos de agua y algunos relieves. No obstante, en el caso de los elementos construidos, aparecen, además de los caminos y la división del suelo, los pueblos, el ferrocarril, los fuertes y las misiones. Cada componente identificado se redibuja; esto permite comprender aisladamente cada componente y se obtienen, por tanto, diversas capas territoriales. Lo que no se logra apreciar eso sí, debido a su escala, son las formas de las edificaciones.

Como tercer paso se identifican repeticiones entre esas morfologías, es decir, se trata de identificar entre dichos elementos patrones o reglas que parecen ser una constante en el territorio cartografiado.

Parte importante de la forma de interpretar los planos se consiguió mediante el redibujo y la manipulación de la cartografía. Esto con el fin de extraer la mayor cantidad de observaciones, o bien, para establecer qué relaciones puede haber sumando factores naturales con elementos construidos. El descubrimiento de algunas relaciones permite la elaboración de una cartografía propia. Ello se hizo cambiando el color de algunos sectores que se consideraron interesantes, simplemente seleccionando unos elementos por sobre otros o enfatizando algunos por sobre otros. Como se puede ver, para entender el porqué de la forma del territorio se hizo un acercamiento a través de la forma misma.

Paralelamente, se buscaron fuentes escritas que ayudasen a explicar el porqué de la forma. Ante aquello resultaron útiles algunas obras (Correa, Molina y Yañez, 2005; Flores, 2012; Guevara, 1902; Leiva, 1984; Navarro, 2008; Pinto, 2010, 2015). Así también, algunas fuentes oficiales desde el Estado durante el período de colonización de la Araucanía (Congreso Nacional de Chile, 1912). El trabajo finalmente acaba siendo el cruce desde ambas fuentes: cartográficas y escritas, pero siempre

\footnotetext{
${ }^{10}$ Estos planos actualmente son bastante usados en el Archivo Regional de la Araucanía como referencia y orientación para fines aclaratorios sobre propiedades que requieren regularizarse o que presentan algún tipo de disputa o asunto no resuelto. La falta de claridad respecto de lo que ocurrió parece continuar hasta ahora.

${ }^{11}$ El documento del censo de 1907 (Comisión central del censo, 1908, p. XXII) señala que la información sobre la cantidad de población indígena que existía antes del censo era incierta y contradictoria. Y que parecía haberse aceptado
}

enfocado a todo lo que respecta a la construcción y la forma física.

\section{Resultados}

\section{Los planos y su descripción: aclaración, orientación y revelación de un territorio desconocido}

Como primera cualidad, los planos de la IGCI revelaron una Araucanía oculta hasta entonces. En esos años la situación era difícil y no había claridad al respecto de todos los sucesos que estaban ocurriendo, sobre todo en el tema de la propiedad ${ }^{10}$. Manifiesta Almonacid $(2009$, p.6) que el asunto de la propiedad de la tierra en la segunda mitad del siglo XIX en el sur de Chile fue complejo y problemático. La situación sobre a quién pertenecía la tierra era poco clara y esto derivó en conflictos.

Estos planos se confeccionaron después de un censo muy importante, el año 1907. Junto a dicho censo fue llevado a cabo un "censo de indios araucanos"11. A partir de Flores y Azocar (2017) se puede plantear que los planos buscan entonces aclarar un problema: cómo descifrar y representar en el espacio los problemas que surgen en la Araucanía. Dichos autores identificaron una relación entre el censo y los planos de la IGCl, y plantean que, en tanto el censo señala "cuántos son", los planos "podían ayudar" a reconocer tanto "dónde están" las personas, así como "de quién es" la propiedad de la tierra. Siguiendo su planteamiento podemos decir que es un plano de aclaración y orientación.

El origen de estos planos al parecer proviene del progreso de los archivos de la Inspección General de Colonización e Inmigración tal como se ve en las memorias correspondientes a los años 1917 y 1918 insertos en la memoria publicada en 1920 :

El progreso del Archivo de esta Inspección, acrecentado con la labor anual de levantamiento de planos i reconocimientos que practican las diversas comisiones de Ingenieros y Agrimensores

que la raza indijena había disminuido (1908, p. XXII). Señala el documento que la cifra 101.118 araucanos corresponde al área entre el Biobío y el golfo de Reloncaví, principalmente en las provincias de Malleco, Cautín y Valdivia. El documento también consigna que la cifra del censo resultó el doble de lo que se había sospechado considerando los cálculos más optimistas (1908, p XXII). En el mismo documento se puede ver la cantidad de datos bastante inexactos acerca de la población indígena estimada que manejaban quienes gobernaban el país. 
desde hace veinticinco años, dio base a la idea de iniciar la formación de las grandes Cartas Catastrales por provincia a escala 1:100 000 . (Inspección General de Colonización e Inmigración, 1920, p.277)

Hasta entonces (1916-1917) según los datos que maneja este trabajo, existían mayoritariamente planos de zonas específicas y acotadas, pero estos dos planos vienen a representar una imagen global y por tanto permiten la articulación de todos los planos pequeños y sueltos. En consecuencia, una característica de los planos es que permite la unión y el entendimiento de todos los mapas anteriores, de los primeros remates, de las primeras colonias, etc.

Los planos vienen a reflejar algo que ya estaba hecho $y$, salvo algunos trazados del ferrocarril, no tiene una vocación proyectual. Es un plano que refleja cosas que ya están. Si bien solo venía a constatar hechos, viene a mostrar algo que no se sabía. Dado su detalle parecen ser cartas que serían bastante útiles para la administración de entonces. Tal como se describe en el mismo documento anteriormente citado:

Estas cartas catastrales, las primeras que se construyen i publican en el país, han sido justamente apreciadas por los servicios incalculables llamados a prestar tanto a las reparticiones públicas como a los particulares. (Inspección General de Colonización e Inmigración, 1920, p.277)

Los planos de la IGCl son documentos que destacan por su minuciosidad, estos están impresos en los documentos correspondientes cada uno a cada provincia ${ }^{12}$. Se representan en escala 1:100.000 y a color. En dicha escala se alcanza a distinguir la medida de 100 metros. En cuanto a los colores se usan solo tres: negro, rojo y azul. Por lo general, el azul designa los cursos de agua, el rojo señala la dimensión de las propiedades rurales y el límite de concesiones, y el resto de los elementos se representan en negro (una muestra se observa en la Figura 2). En términos generales los planos nos muestran una gran cantidad de datos. En primer lugar, revelan criterios de gobierno y nos permiten ver las formas administrativas de la Araucanía de entonces, permiten distinguir las provincias, departamentos, subdelegaciones y también el límite internacional con Argentina. Todas estas líneas de límite se dibujan con diferentes tipologías de líneas segmentadas.

Dentro de los elementos construidos que aparecen en los planos destacan los caminos, algunos puentes, fuertes militares, algunas misiones, el trazado del ferrocarril tanto en explotación como en proyecto y las estaciones de esas líneas tanto en zonas rurales como dentro de los pueblos. También se aprecia la forma general de los pueblos, donde se pueden observar que en todos prima la tipología de manzana cuadriculada, pero con diferentes orientaciones, además la escala permite ver dónde están los espacios vacíos o plazas, también se observan zonas de cuadrículas marcadas, pero sin rellenar. La escala de los planos también permite observar, con un grado importante de realidad, pero sin plena precisión, la forma de la división del suelo rural.

\footnotetext{
${ }^{12}$ Nos encontramos con estos planos en tres versiones. La primera, la que más hemos usado, son las imágenes que dispone el Archivo Regional de la Araucanía en formato digital. La segunda fuente la constituyen los documentos originales disponibles en el Archivo Nacional de Chile (Figuras 1 y 2 ), elaborados en un formato de 32 x 49 centímetros a modo de libro; dentro de ellos las láminas interiores se extienden y alcanzan una medida, por lámina, de 59,5 x 48,2 cm para Malleco, y 48,4 x $58 \mathrm{~cm}$ para Cautín. Malleco
}

se divide en doce láminas y Cautín en quince. La tercera fuente es la unidad de catastro de la SEREMI de Bienes Nacionales de la Araucanía (Figura 3), que se encuentra sobre tela y con todas las láminas unidas, por provincia. La provincia de Malleco alcanza una dimensión de 121 x $195 \mathrm{~cm}$ aproximadamente. La provincia de Cautín, en tanto, alcanza una dimensión de $250 \times 129 \mathrm{~cm}$ aproximadamente. Consideramos que todos los documentos son fruto de distintos ejemplares del mismo documento en escala 1:100.000. 


\section{Figura 1}

Imagen de un ejemplar de la carta catastral de la provincia de Malleco

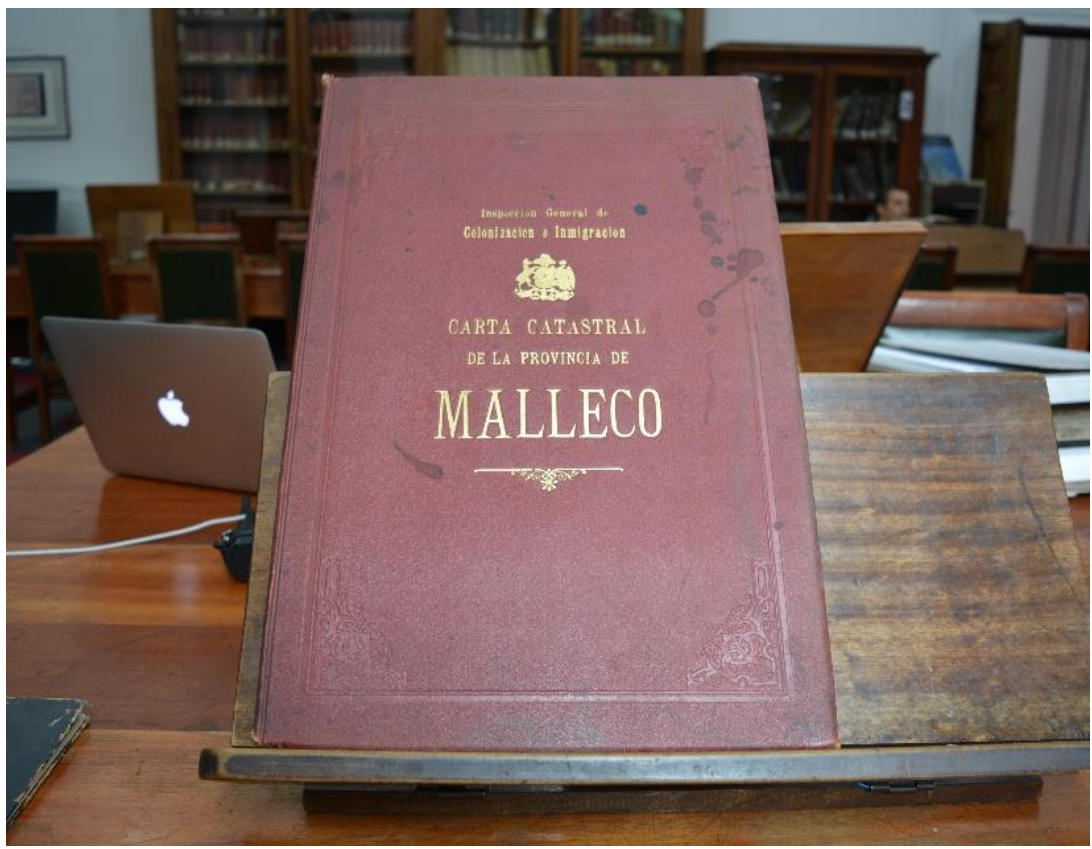

Fuente: Fotografía del autor de un ejemplar disponible en Archivo Nacional de Chile.

\section{Figura 2}

Imagen de una porción de una de las láminas que compone la carta catastral de Cautín

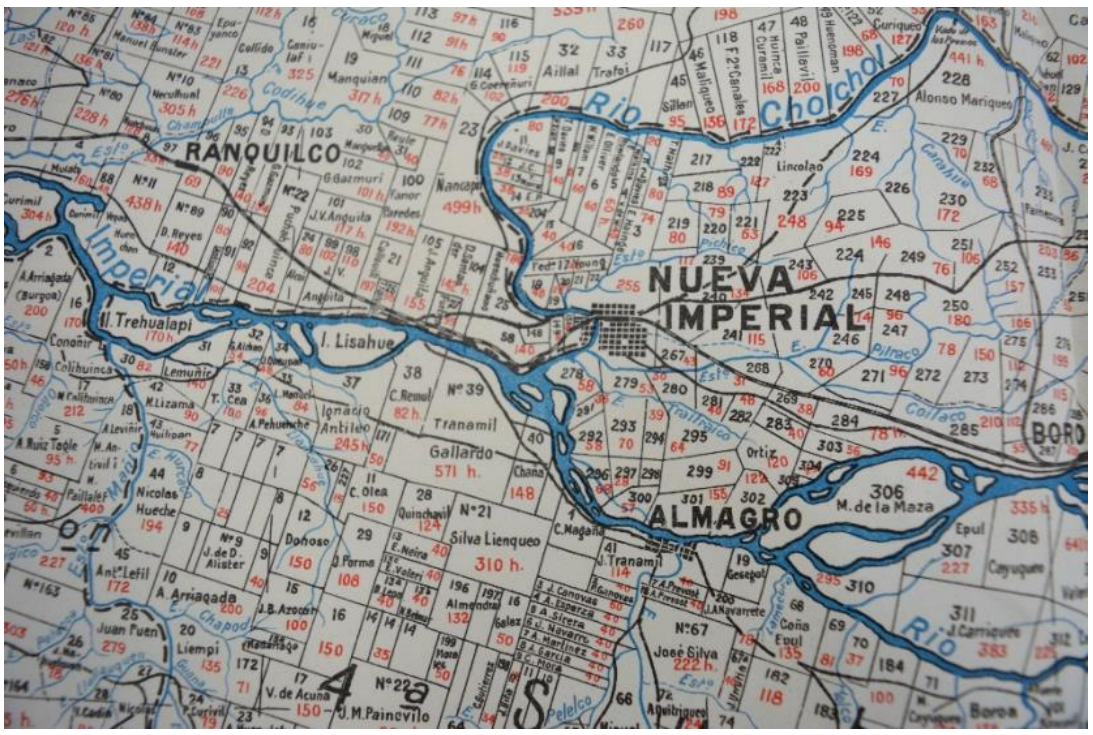

Fuente: Fotografía del autor del ejemplar disponible en Archivo Nacional de Chile. 


\section{Figura 3}

Fotografía de la carta catastral de Cautín con todas sus láminas unidas

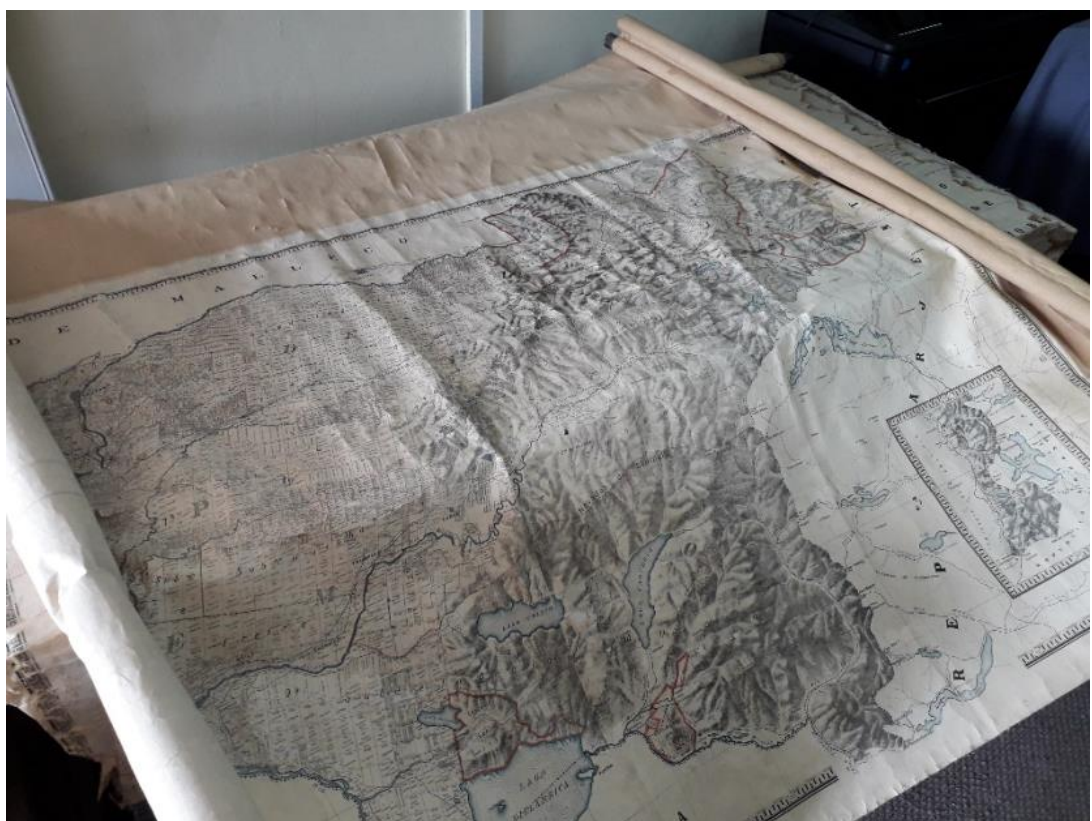

Fuente: Fotografía del autor del ejemplar disponible en SEREMI Araucanía de Bienes Nacionales de Chile.

Además de los elementos construidos, los planos revelan gran cantidad de elementos naturales. Los planos son detallados en los cursos de aguas, mostrando también varios vados. Normalmente los cursos de agua se dibujan a una sola línea, pero en ocasiones se dibujan a doble línea y se aprecian los anchos de algunos cursos de agua importantes. Al considerar los cursos de agua en el trazado, los planos muestran la mayoría de los puntos bajos marcados y muchos de estos cursos de agua inciden en la forma de dividir el suelo. En cuanto a los puntos altos, hay menos información, aunque se pueden ver algunos relieves importantes (usando normales para su representación) y algunos puntos altos que determinaron la división del suelo rural. Así también se ven varios pasos cordilleranos.

La cantidad de datos que presentan los planos de la IGCI revela qué fue lo más importante según la visión de los colonizadores y de la institucionalidad estatal. Hay valores que se realzaron por sobre otros. Debido a su escala, la forma de la división del suelo parece ser la mayor aportación de estos documentos. La parcela o propiedad rural es lo que más llama la atención porque parecen procesos más diversos y complejos, en cambio las plantas de los pueblos parecen ser más homogéneas.

Es necesario señalar que la constitución de la propiedad fue un proceso determinante para la conformación espacial de la Araucanía. Es posible observar la morfología general de las propiedades y toda la complejidad del proceso de división del suelo. Se observa que buena parte de las hijuelas o parcelas contiene el número de sitio o lote, el dueño (sean personas naturales, fundos o sociedades) y el número de hectáreas. Siendo esos los datos se puede observar dos tipos de propiedades en base al tipo de adjudicatario: tierras mapuches y tierras no mapuches. Dentro de las tierras mapuches se ven buena parte de los títulos de merced entregados en el proceso de colonización chileno, aunque no todos puesto que dicho proceso acaba recién el año 1929 (Almonacid, 2008, p.115). Dentro de las tierras no mapuches se observan los diferentes modos de 
entrega $^{13}$ que se ejecutaron entonces (tierras para colonias de extranjeros, remates, concesiones, etc.). Con todos estos detalles se convierte en una imagen bastante precisa de la Araucanía de entonces en sus dos provincias Cautín y Malleco.

Respecto a las tipologías morfológicas de la división del suelo, vemos que son bastante diversas, existiendo patrones que se acumulan en ciertas áreas, pero no en toda la dimensión de las provincias. Esa división del suelo no tiene una concordancia con otras construcciones, al menos no con el ferrocarril ni con la red urbana.

\section{Las repeticiones que revelan los planos de la IGCI}

Los planos contienen muchos datos, como ya se mencionó, y una de las características más significativas (morfológicamente hablando) es que muchos de sus componentes manifiestan estructuras repetitivas. Planteamos que los planos de Boloña son una suma de repeticiones que nos pueden revelar las voluntades que influyeron tras la constitución del espacio que fabricó el Estado de Chile.

Estas repeticiones son más evidentes en la división del suelo. Los planos muestran una gran variedad de formas y tamaños parcelarios (Figura 4) y, como no es fácil descifrarlos en su conjunto, es necesario acudir a las repeticiones. Se puede ver que esta diversidad también se debió a la diversidad de formas administrativas de entrega de tierras.

Es posible, no obstante, observar de forma bastante general, solo dos tipos de líneas o trazados: la línea recta o abstracta (que siempre aparece delineada en los planos) y la línea que se ajusta a algún accidente natural (por lo general cursos de agua y a veces caminos, la que no siempre aparece explícitamente delineada, pero se deduce). El agrimensor contó con esas dos herramientas geométricas para trazar la división del suelo en la Araucanía.

También los planos revelan una serie de condicionantes o criterios que generan repeticiones. Un primer condicionante es el norte magnético. Se observa una orientación constante hacia el norte magnético que se repite en casi todas las agrupaciones de parcelaciones ortogonales. Un segundo condicionante es la forma de dividir a través de grandes franjas o fajas, de río a río. Aquí la regla constante es el ancho de las propiedades, en algunos casos es 2000 metros. Un tercero, serían los puntos bajos o cursos de aguas; en algunas parcelaciones de colonias se puede ver que los pequeños cursos de agua fueron marcando pequeñas parcelas alargadas que en sus extremos colindaban con cursos de aguas. Un cuarto, son algunos puntos altos, los que se utilizaron como puntos de referencia, y desde donde se trazaron líneas.

Algunas normativas ayudan también a la explicación de las repeticiones o tipologías. Una de las leyes más importantes es la del 4 de diciembre de 1866 (Congreso Nacional de Chile, 1912, pp. 6-9), que dio una pauta muy significativa: establece que, para los deslindes de las propiedades mapuches, en lo posible, se considerarán elementos naturales. Esta es una regla que se aplica en buena parte de los títulos de merced, aunque no se aplicó a todos de forma taxativa. Así mismo dicha ley establece una medida, ya que prohibía rematar terrenos más allá de un tamaño de 500 hectáreas, dicha medida parece haber dado lugar a un tamaño parcelario de $2000 \times 2500$ metros, que se repite bastante ${ }^{14}$.
13 El trabajo de Correa, Molina y Yáñez (2005) ha sido relevante para identificar y comprender esos modos de entrega de tierras.

\footnotetext{
${ }^{14}$ Hay que considerar que, eso sí, en varios casos el tamaño que adquirieron algunos fue mayor a 500 hectáreas.
} 


\section{Figura 4}

Redibujo del parcelario del plano de la IGCl en la zona de Malleco donde se aprecian diferentes repeticiones morfológicas en la división del suelo

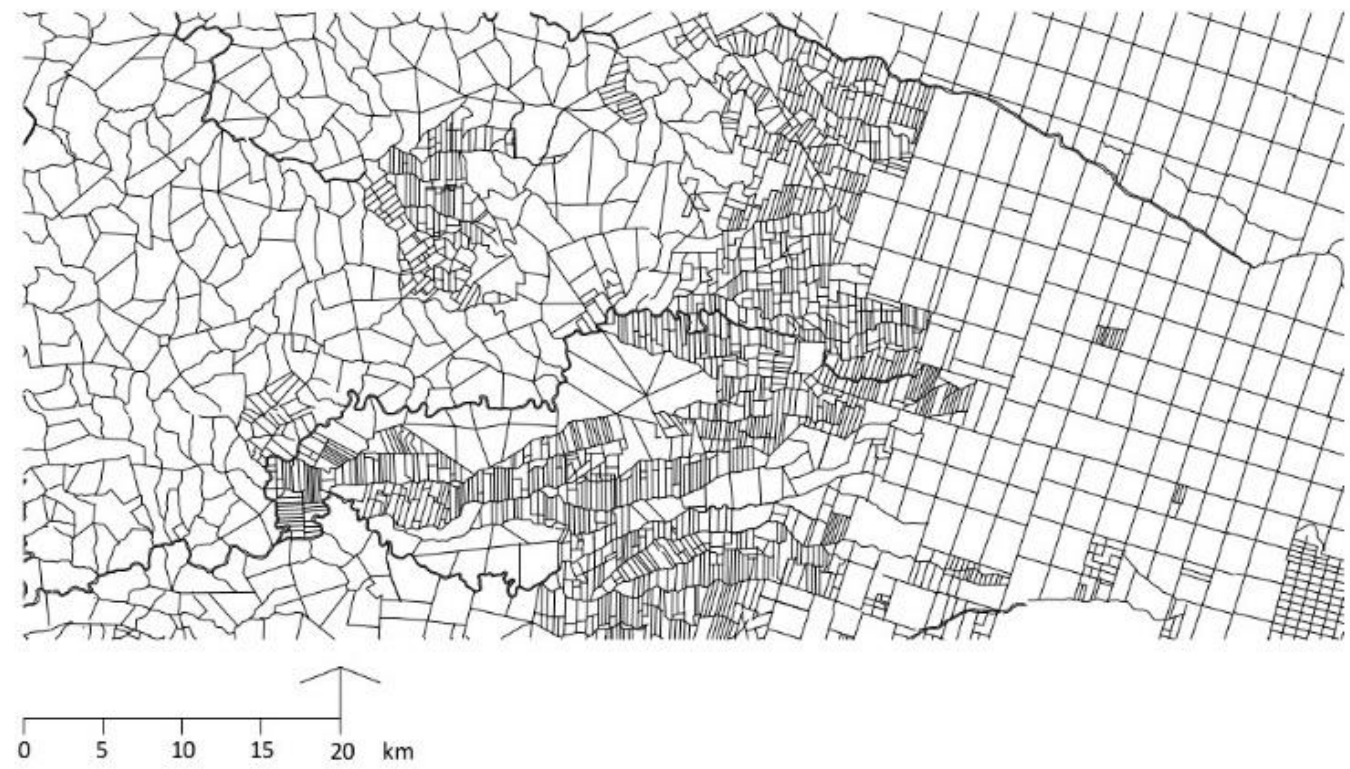

Fuente: Elaboración propia sobre los planos de la ICGI en digital proporcionados por el Archivo Regional de la Araucanía.

\section{La continuidad norte-sur del país que se construye}

Los planos de la IGCI permiten reflejar las acciones físicas que el Estado ejerció en el territorio y así también la disposición espacial de esas acciones. Dichas acciones se tradujeron a los componentes del plano, en el presente caso se consideraron los fuertes, la división del suelo, la línea del ferrocarril y los primeros pueblos. La disposición de estos componentes refleja la idea de una estructura espacial que busca la continuidad del país en la dirección norte a sur.

Si se pone atención a los componentes del plano se observa que morfológicamente no encajan muy bien unos con otros porque, se debe recordar, Nicanor Boloña elaboró estos planos en base a una serie de planos fragmentados de áreas más pequeñas en extensión y que ya existían entonces. Este desencaje territorial también se observa entre la forma urbana y la forma de la parcela o propiedad rural. Ahora bien, pese a que se aprecian estos desencajes, en la gran escala se pueden divisar formas que hablan de intenciones espaciales sobre el territorio. Para esto es necesario considerar ambos planos en conjunto, tanto el de Malleco como el de Cautín, de otro modo es imposible descubrir esta cualidad de continuidad nortesur que tiene el territorio. Asimismo, es necesario relacionar los componentes mencionados.

Son las repeticiones que se acumulan en vastas áreas y la relación entre los componentes identificables las que nos dan unas ideas espaciales en la gran escala ${ }^{15}$. Si consideramos la repetición y acumulación de las dos principales tipologías de las propiedades o hijuelas a nivel general, observamos, en la escala regional, propiedades con una tendencia a la ortogonalidad y propiedades con unas tipologías más orgánicas. Considerando estas dos tipologías vemos que se van formando agrupaciones que generan una imagen, y vemos que toda la división del suelo adquiere una forma tal como en la Figura 5 donde las propiedades con formas más orgánicas generan un

${ }^{15}$ Consideramos que en la pequeña escala también se encuentran algunas reglas, pero aquello es tema de otro trabajo. 
área o cuerpo central (marcado en rojo) con una tendencia alargada norte-sur ${ }^{16}$.

Cuando se relacionan a este esquema del cuerpo central, la ubicación de los primeros fuertes, de los pueblos y el ferrocarril se revela una lógica (igura 5 ). Este cuerpo es lo que estructura el avance de norte hacia el sur y que se relaciona con la estructura del ferrocarril. Se puede plantear, por tanto, que estos cuatro procesos mencionados se superponen unos a otros. Si consideramos los antecedentes históricos vemos que el primer acto son los avances de fuertes militares a la par de un incipiente inicio de urbanización, luego la división del suelo con unas primeras subastas o remates $y$, como proceso final, la construcción del ferrocarril (Antivil, 2018). El orden de estos procesos no es completamente sucesivo uno hacia otro. Por ejemplo, los procesos de avance militar todavía no acababan cuando ya se empieza a dividir el suelo en las zonas conquistadas y, asimismo, cuando ya habían acabado de fundarse la mayoría de los pueblos la división del suelo aún no acababa. De hecho, los planos de la IGCl manifiestan que no toda la tierra estaba aún dividida.

\section{Figura 5}

Redibujo de los dos planos de la IGCI juntos (Malleco y Cautín)

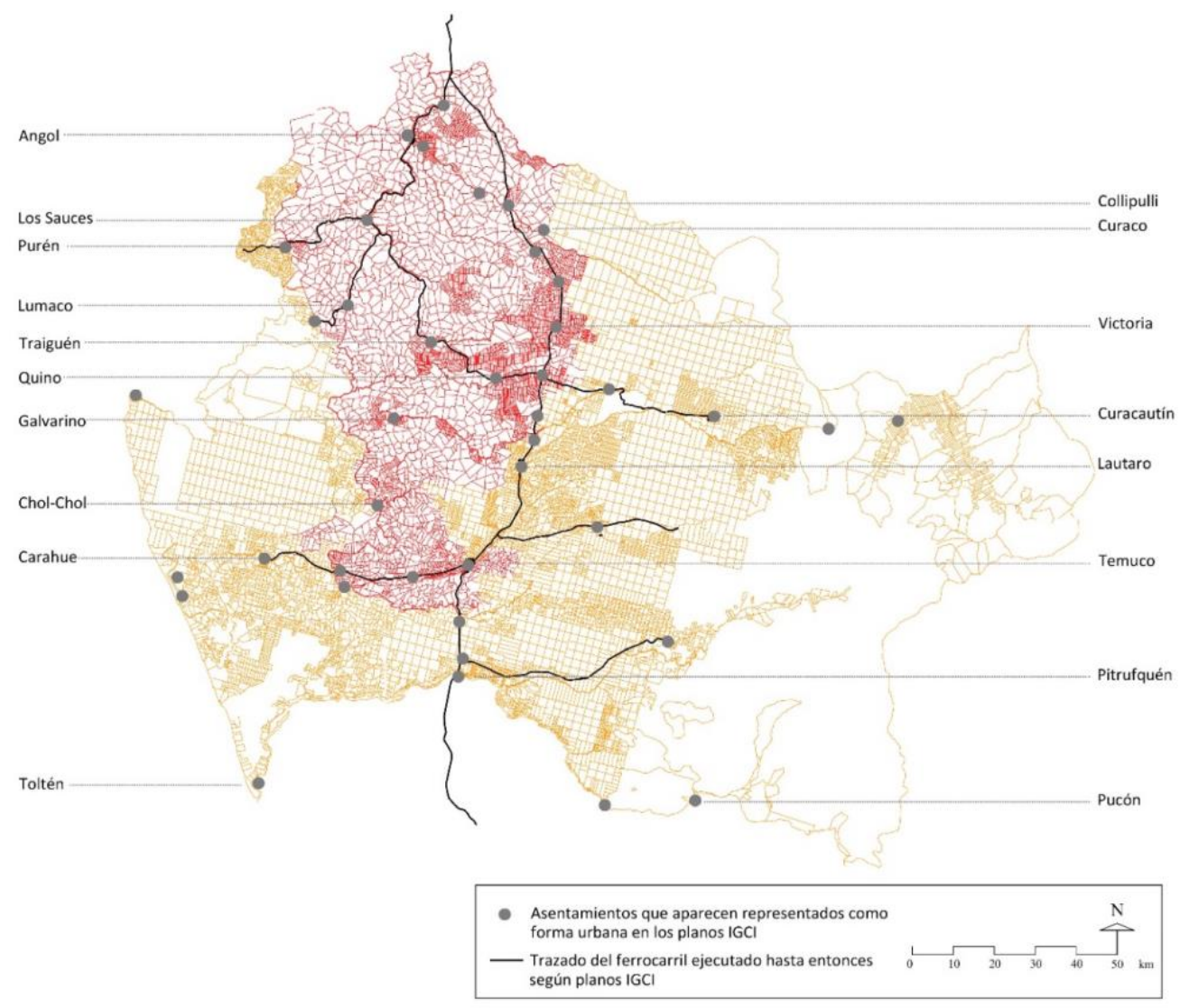

Nota: Se han sumado las capas relativas a la división del suelo rural (diferenciando las que tienen tendencia a seguir repeticiones ortogonales [en color naranja] de las irregulares [en color rojo]), ferrocarril y asentamientos urbanos. Hemos añadido los nombres de algunos pueblos para orientarse.

Fuente: Elaboración propia sobre planos de la IGCl en digital proporcionados por el Archivo Regional de la Araucanía.

${ }^{16}$ La idea de parcelas o propiedades rurales con formas orgánicas frente a ortogonales, el cuerpo central y de la continuidad norte sur también ya aparece en el trabajo de Antivil (2018), aunque la última está menos enfatizada. 
Si bien no fueron sucesivos y ordenados, en todos estos procesos se ve el mismo principio: un avance principalmente desde el norte hacia el sur. Para esto hay que observar otras fuentes además de los planos de la IGCI. Por un lado, según se desprende de los escritos de Navarro (2008), los fuertes y primeros asentamientos que se ubicaron en la zona de la depresión intermedia se fueron instalando de tal modo de seguir el curso de algunos ríos de norte a sur. Por otro lado, en el plano de unos primeros remates hecho en 1888 (Figura 6), si se observan los años de las zonas que se remataron, se ve que las ubicadas en la zona de Malleco son más antiguas que las aledañas a la zona del Cautín.

\section{Figura 6}

Plano que refleja los primeros remates hechos en la Araucanía, año 1888.

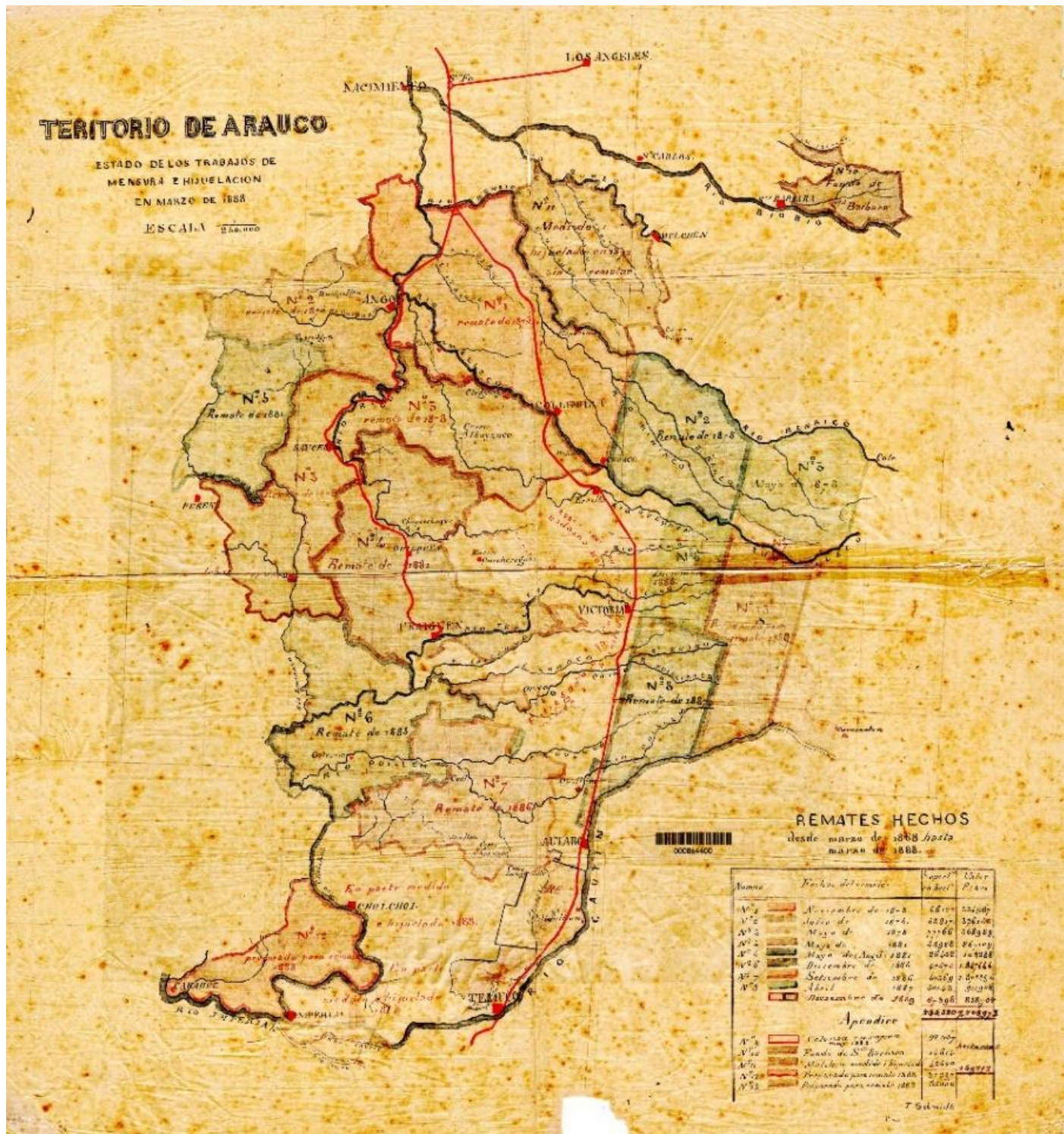

Nota: Título del plano: Territorio de Arauco. Estado de los trabajos de mensura e hijuelación en marzo de 1888. Escala del plano original 1:250.000. Parece estar firmado por T. Schmidt. Fuente: Archivo de planos del Ministerio de Bienes Nacionales de Chile 
En otras cartografías que aparecen en el trabajo de Rosenblitt y Sanhueza (2010, pp. 208-209) se puede ver también la idea de continuidad norte-sur, las cuales, al parecer, son previas al proceso de división del suelo. Se encuentran la elaborada por Tirso Rodríguez titulada "Croquis del valle central de la Araucanía con los caminos seguidos por las divisiones que lo han cruzado", y otro mapa que refleja algunos fuertes y caminos titulado "Croquis de la Línea del Malleco i nuevos fuertes de Cautín". Dichas cartografías evidencian la importancia de la depresión intermedia que corre entre Angol y Temuco. La forma del territorio natural no permitía muchas variaciones para colonizar. El ferrocarril en toda esta sucesión de construcciones viene a ser el elemento más relevante puesto que vendrá a consolidar la visión espacial norte-sur del país que se quiere construir.

Los planos de la IGCI revelan una idea que no es del todo original, que ya se ve desde los primeros conquistadores junto a Pedro de Valdivia (Garretón, 1997). El recorrido que hicieron para conocer el país fue, precisamente, de norte a sur principalmente, y en esa dirección fueron fundando asentamientos y estableciendo dominio sobre el territorio ${ }^{17}$. Es necesario señalar que con la derrota en Curalaba la frontera de la Colonia se instala principalmente al norte del Biobío, y también en otros puntos al sur de la actual Araucanía, de manera que se estableció un territorio fragmentado y sin continuidad. Esta cualidad de un país desconectado es lo que, probablemente, hace que ya en el siglo XVIII aparezcan ideas que buscan dominar la Araucanía generando una continuidad territorial norte-sur ${ }^{18}$.

La cualidad norte-sur también se ve tanto en las cartografías de la Colonia como en la que se hace durante la República de Chile y en los primeros planos militares que se hacen con objeto de conquistar el territorio (Rosenblitt y Sanhueza, 2010). Esto sin duda genera una imagen de país y una predisposición a determinado modo de construir el territorio y también de homogeneizarlo ${ }^{19}$.

\section{Discusión}

La Araucanía ha sido investigada por diversas disciplinas, en ese sentido los hallazgos de este trabajo han sido una prueba del valor que tiene el método de algunos arquitectos que estudian la forma y el dibujo del territorio, y como este puede ser válido tal como otras disciplinas que lo describen y estudian. Entre las lecciones que nos deja este método es que, en la medida que se dibuja y redibuja el plano, se comprenden mejor sus problemas e identidades. El dibujo del plano identifica componentes y evidencia repeticiones y reglas, las cuales evidencian intenciones y diversos procesos. Otro valor del método es que es aplicable a otros lugares aportándoles un conocimiento o nuevas luces que antes no se habían logrado concretar. El método, por último, ha permitido generar nueva cartografía de la Araucanía a partir de los propios planos ICGI, como se manifiesta en la Figura 5.

Los hallazgos también han demostrado que los planos de la IGCI son una herramienta valiosa para investigar. En ese sentido se considera que la relación entre historia y la representación del espacio es un campo interesante para futuros trabajos.

La metodología planteada ha sido útil, si bien no posee un nombre ni reglas en extremo específicas, sino que es fruto del trabajo y convicción de algunos arquitectos con intereses sobre el territorio. Dicha metodología está permitiendo continuar los conocimientos que se tienen acerca de este territorio, especialmente dando algunas luces respecto al porqué de la forma del territorio. Hoy la Araucanía sigue siendo una región que plantea realidades sin claridad e irresueltas. Mucho se discute sobre este territorio mediante trabajos y documentos escritos, pero no son muchos trabajos los que consideran el valor del
${ }^{17}$ Si bien establece un punto en Villarrica pensando en la comunicación hacia el lado oriental de la cordillera, consideramos que, al principio de su proceso colonizador, prevalece su visión de un país largo norte-sur.

${ }^{18}$ Ya desde el siglo XVIII la Araucanía es pensada por autoridades como un territorio a "proyectar" o a "integrar". Señala Gándara (2016, p. 63) que "durante los siglos coloniales el estado de Arauco fue sometido a diferentes estrategias de integración. La acción bélica, las misiones, el comercio y los parlamentos fueron los medios más utilizados para incorporar y asimilar este territorio a los cánones culturales y políticos españoles." Dicha autora nombra (2016:69) a algunos intelectuales que pensaron la Araucanía y en dicho

\footnotetext{
trabajo vemos que era importante para los proyectistas mencionados el tema de la continuidad territorial y física que debía tener el gobierno colonial, el cual estaba interrumpido por las agrupaciones mapuches.

${ }^{19}$ Un aspecto a considerar es que esta condición espacial norte-sur que se buscaba imponer llegó a pasar por alto la cualidad espacial en la posición esteoeste conformada por los ríos y sus cuencas, aspecto interesante planteado por Núñez (2012).
} 
espacio y su representación planimétrica o cartográfica como insumo valioso para elaborar tesis precisas.

El trabajo abre y sigue poniendo de manifiesto, junto con otros trabajos que tratan estos mismos temas, la importancia que tiene este proceso de intervención estatal a gran escala llevado por Chile desde la segunda mitad del siglo XIX sobre el territorio de la Araucanía.

\section{Conclusiones}

Los planos de la $\mathrm{IGCl}$ poseen valores importantes. En primer lugar, porque aclaran y aportan gran cantidad de datos respecto del proceso de construcción territorial chileno sobre la Araucanía en el siglo XIX. En segundo lugar, porque al examinar los planos se aprecian voluntades proyectuales, es decir, es un mapa que permite ver criterios de construcción que hablan de criterios territoriales, especialmente la idea de estructurar un país con una columna vertebral territorial norte sur.

Los planos entregan luces importantes para un momento en que todo era confuso y con conflictos sobre la propiedad de la tierra tal como lo han señalado algunas fuentes. La pulcritud y detalle de los planos permite seguir comprendiendo hoy acerca de lo que ocurrió a nivel físico y constructivo en la colonización de la Araucanía. Los planos buscaron ser un registro de lo ocurrido desde la visión de la entidad colonizadora y permiten ver qué temas eran los que más le interesaban, en ese sentido parece ser que la forma de la propiedad rural era uno de ellos. Los planos además permiten distinguir componentes territoriales, como factores naturales y elementos construidos, lo cual permite someterlo a un estudio morfológico siguiendo los métodos descritos.

Cuando se han identificado algunos componentes se observa la importancia que tienen las repeticiones de estos para entender la forma de los planos. La acumulación de repeticiones genera grandes morfologías legibles en la gran escala y por esa razón también es posible establecer ideas e intenciones espaciales a ese nivel. Las repeticiones morfológicas también se explican por normativas desde el Estado que debían seguir quienes construyeron el territorio, en ocasiones también por reglamentos propios, o en modos de operar o prácticas no escritas propias de la profesión u oficio.

A pesar de algunos desencajes en cuanto a la forma se puede ver la idea de una continuidad norte-sur. Pero esto solo es posible observarlo con los dos planos IGCI en conjunto. Para observar la continuidad, es necesario, además de las repeticiones, relacionar la división del suelo con las otras construcciones asociadas (ferrocarril y ciudades). La continuidad norte-sur además se manifiesta en la construcción de los fuertes e incluso en mapas más antiguos e ideas ya presentes en el país.

Se ha hallado que la forma resultante de la construcción de la Araucanía es, muy probablemente, reflejo de la influencia de cartografías e ideas preexistentes ya que, como se señaló, la idea de un país alargado y continuo es una constante en algunas cartografías previas a la ocupación de la Araucanía [B]

\section{Referencias bibliográficas}

Almonacid, F. (2008). La división de las comunidades indígenas del sur de Chile, 1925-1958: un proyecto inconcluso. Revista de Indias, 68(243), 115-150. https://doi.org/https://doi.org/10.3989/revindias.200 8.i243.649

Almonacid, F. (2009). El problema de la propiedad de la tierra en el sur de Chile (1850-1930). Historia, 42(1), 5-56. https://dx.doi.org/10.4067/S0717$\underline{71942009000100001}$
Álvarez, L. (2000). Cartografía y geodesia: las innovaciones de la Oficina de Mensura de Tierras de Chile a principios del siglo XX (1907-1914). Scripta Nova. Revista Electrónica de Geografía y Ciencias Sociales, 4(69). https://revistes.ub.edu/index.php/ScriptaNova/articl e/view/220

Antivil, W. (2018). Dibujando la Araucanía: la construcción, la forma y el dominio de un territorio (Tesis de doctorado). Universitat Politècnica de Catalunya, Barcelona, España. https://www.tdx.cat/handle/10803/462096 
Bisbal-Grandal, I. (2019). El paisaje araucano en la cartografía de 1575 a 1646: Una imagen del territorio basada en el dominio. AUS [Arquitectura / Urbanismo / Sustentabilidad], (26), 4-9. https://doi.org/10.4206/aus.2019.n26-02

Capellà, H. (2009). Por los caminos de la identidad y del desarrollo regional. Atenea, (500), 75-90.

http://dx.doi.org/10.4067/S0718$\underline{04622009000200006}$

Chamorro, C. y Urbina, S. (2018). Expresión cartográfica y atributos urbanísticos de Valdivia y Nueva Imperial (sur de Chile): un análisis comparado. Revista de Urbanismo, (39), 1- 16. https://doi.org/10.5354/07175051.2018.48762

Comisión central del Censo. (1908). Memoria: presentada al Supremo gobierno por la comisión del censo. Santiago de Chile: Universo. http://www.memoriachilena.gob.cl/602/w3-article8117.html

Congreso Nacional de Chile. (1912). Comisión Parlamentaria de Colonización. Informe, proyectos de ley, actas de sesiones y otros antecedentes. Santiago de Chile: Sociedad "Imprenta y Litografía Universo." Recuperada de https://obtienearchivo.bcn.cl/obtienearchivo?id=doc umentos/10221.1/22193/5/125970.pdf

Correa, M., Molina, R. y Yáñez, N. (2005). La Reforma Agraria y las tierras mapuches: Chile 1962 - 1975. Santiago de Chile: LOM Ediciones.

De Solá-Morales, M. y Parcerisa, J. (1981). La forma d’un país. Quaderns d'Arquitectura i Urbanisme, (número extra 1), 4-13.

Eizaguirre, X. (2019). El territorio como Arquitectura. Barcelona: Laboratori d'Urbanisme de Barcelona.

Figueroa, C. (2011). Geografías en disputa: La construcción del Chile territorial. Revista 180, (27). http://dx.doi.org/10.32995/rev180.Num27.(2011).art-121

Flores, J. (2012). La Araucanía y la construcción del sur de Chile, 1880-1950. Turismo y vías de transporte. Scripta Nova. Revista Electrónica de Geografía y Ciencias Sociales, 16(418). https://revistes.ub.edu/index.php/ScriptaNova/articl e/view/14800

Flores, J. y Azócar, A. (2017). Mapas para el Estado. La representación de la Araucanía: 1836 -1916. Scripta Nova. Revista Electrónica de Geografía y Ciencias Sociales, 21(562). https://doi.org/10.1344/sn2017.21.18344
Gándara, N. (2016). Representaciones de un territorio. La frontera mapuche en los proyectos ilustrados del Reino de Chile en la segunda mitad del siglo XVIII. Historia Crítica, (59), 61-80. https://doi.org/10.7440/histcrit59.2016.04

Garretón, J. (1997). El urbanismo en Chile: Conquista y Colonia. Concepción: Ediciones Universidad de Concepción.

González-Cebrián, J. (1984). La Ciudad a través de su plano: la Coruña. La Coruña: Ayuntamiento.

González, J. y Bernedo, P. (2013). Cartografía de la transformación de un territorio: La Araucanía 18521887. Revista de Geografía Norte Grande, (54), 179198. http://dx.doi.org/10.4067/S071834022013000100010

González, J. (2007). Primeros levantamientos cartográficos generales de Chile con base científica: los mapas de Claudio Gay y Amado Pissis. Revista de Geografía Norte Grande, (38), 21-44. http://dx.doi.org/10.4067/S071834022007000200002

González, H. y Llancavil, D. (2017). La reconstrucción de un espacio de poder a través de los mapas. El caso de la cartografía misional del Obispado de Villarrica, Chile (1890-1935). HiSTOReLo. Revista de Historia Regional y Local, 9(17), 380-405. http://www.scielo.org.co/scielo.php?script=sci arttex t\&pid=S2145-

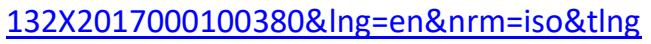

Gregotti, V. (1972). El Territorio de la arquitectura. Barcelona: G. Gili.

Guevara, T. (1902). Historia de la civilización de Araucanía. Tomo III. Los Araucanos y la República. Santiago de Chile: Imprenta, litografía i encuadernación Barcelona.

Hidalgo, G., Rosas, J. y Strabucchi, W. (2012). La representación cartográfica como producción de conocimiento. Reflexiones teóricas en torno a la construcción del plano de Santiago de 1910. ARQ (Santiago), (80), 62-75.

http://dx.doi.org/10.4067/S0717$\underline{69962012000100016}$

Inspección General de Colonización e Inmigración. (1920). Memoria de la Inspección Jeneral de Colonización e inmigración correspondiente a los años 1915, 1916, 1917 y 1918. Santiago de Chile: Imprenta i encuadernación "Universidad." [Biblioteca Nacional de Chile] 
Instituto Geográfico Militar y Medina, J. T. [autores secundarios] (1952). Cartografía hispano colonial de Chile. Il Atlas: homenaje del Ejército de Chile a José T. Medina. Santiago de Chile: Instituto Geográfico Militar.

Leiva, A. (1984). El primer avance a la Araucanía: Angol 1862. Temuco: Ediciones Universidad de la Frontera.

Navarro, L. (2008). Crónica militar de la conquista y pacificación de la Araucanía. Desde el año 1859 hasta su completa incorporación al territorio nacional. Santiago de Chile: Pehuén editores.

Núñez, A. (2012). El país de las cuencas: fronteras en movimiento e imaginarios territoriales en la construcción de la nación. Chile siglos XVIII-XIX. Scripta Nova. Revista Electrónica de Geografía y Ciencias Sociales, 16(418).

https://revistes.ub.edu/index.php/ScriptaNova/articl e/view/14803

Pinto, J. (2010). Ignacio Domeyco. Viaje a la Araucanía en el año 1845 y otros documentos sobre la Frontera. En I. Domeyko, La Araucanía y sus habitantes: Recuerdo de un viaje hecho en las provincias meridionales de Chile, en los meses de enero y febrero de 1845 (pp. ixIviii). Santiago de Chile: Cámara Chilena de la Construcción, Pontificia Universidad Católica de Chile, Dirección de Bibliotecas Archivos y Museos.
Pinto, J. (2015). La formación del Estado, la nación y el pueblo mapuche. De la inclusión a la exclusión. Temuco: Universidad de la Frontera.

Rojo, F. y Hernández, J. (2019). Colonización y nuevo territorio: la formación de la elite comercial de Temuco, 1885-1913. Revista de Geografía Norte Grande, (73), 185-209. https://doi.org/https://dx.doi.org/10.4067/S0718$\underline{34022019000200185}$

Rosas, J., Hidalgo, G., Strabucchi, W. y Bannen, P. (2015). El Plano Oficial de Urbanización de la Comuna de Santiago de 1939: Trazas comunes entre la ciudad moderna y la ciudad preexistente. ARQ (Santiago), (91), 82-93. http://dx.doi.org/10.4067/S071769962015000300013

Rosenblitt, J. y Sanhueza, C. (2010). Cartografía histórica de Chile, 1778-1929. Santiago de Chile: Cámara Chilena de la Construcción, Pontificia Universidad Católica de Chile, Dirección de Bibliotecas Archivos y Museos.

\section{Cartografías}

Inspección General de Colonización e Inmigración (1916). Carta general de colonización de la provincia de Cautín [Material cartográfico]. Formada con los datos del archivo de la Inspección General de Colonización e Inmigración. Construida i dibujada por Nicanor Boloña. Escala 1:100.000. Documento digitalizado. Archivo regional de la Araucanía, Temuco, Chile.
Inspección General de Colonización e Inmigración (1917). Carta general de colonización de la provincia de Malleco [Material cartográfico]. Formada con los datos del archivo de la Inspección General de Colonización e Inmigración. Construida i dibujada por Nicanor Boloña. Escala 1:100.000. Documento digitalizado. Archivo regional de la Araucanía, Temuco, Chile. 